\title{
Quantification of upper limb kinetic asymmetries in front crawl swimming
}

\author{
Pedro G. Morouço ${ }^{\mathrm{a}, \mathrm{b}, *}$, Daniel A. Marinho ${ }^{\mathrm{b}, \mathrm{c}}$, Ricardo J. Fernandes ${ }^{\mathrm{d}, \mathrm{e}}$, \\ Mário C. Marques ${ }^{\mathrm{b}, \mathrm{c}}$ \\ ${ }^{a}$ Centre for Rapid and Sustainable Product Development, Polytechnic Institute of Leiria, Portugal \\ ${ }^{\mathrm{b}}$ Research Centre in Sport, Health and Human Development, Covilhã, Portugal \\ ${ }^{\mathrm{c}}$ Department of Sport Sciences, University of Beira Interior, Covilhã, Portugal \\ ${ }^{\mathrm{d}}$ Centre of Research, Education, Innovation and Intervention in Sport, Faculty of Sport, University of Porto, Porto, Portugal \\ ${ }^{\mathrm{e}}$ Porto Biomechanics Laboratory, University of Porto, Porto, Portugal
}

\section{A R T I C L E I N F O}

Article history:

\section{Keywords:}

Symmetry

Force

Training

Performance

\begin{abstract}
A B S T R A C T
This study aimed at quantifying upper limb kinetic asymmetries in maximal front crawl swimming and to examine if these asymmetries would affect the contribution of force exertion to swimming performance. Eighteen high level male swimmers with unilateral breathing patterns and sprint or middle distance specialists, volunteered as participants. A load-cell was used to quantify the forces exerted in water by completing a $30 \mathrm{~s}$ maximal front crawl tethered swimming test and a maximal $50 \mathrm{~m}$ free swimming was considered as a performance criterion. Individual force-time curves were obtained to calculate the mean and maximum forces per cycle, for each upper limb. Following, symmetry index was estimated and breathing laterality identified by questionnaire. Lastly, the pattern of asymmetries along the test was estimated for each upper limb using linear regression of peak forces per cycle. Asymmetrical force exertion was observed in the majority of the swimmers (66.7\%), with a total correspondence of breathing laterality opposite to the side of the force asymmetry. Forces exerted by the dominant upper limb presented a higher decrease than from the non-dominant. Very strong associations were found between exerted forces and swimming performance, when controlling the isolated effect of symmetry index. Results point that force asymmetries occur in the majority of the swimmers, and that these
\end{abstract}

\footnotetext{
* Corresponding author at: Polytechnic Institute of Leiria, Centre for Rapid and Sustainable Product Development (CDRSP), Centro Empresarial da Marinha Grande, Rua de Portugal, Zona Industrial, 2430-028 Marinha Grande, Portugal. Tel.: +351 910079444.

E-mail address: pedro.morouco@ipleiria.pt (P.G. Morouço).
} 
asymmetries are most evident in the first cycles of a maximum bout. Symmetry index stood up as an influencing factor on the contribution of tethered forces over swimming performance. Thus, to some extent, a certain degree of asymmetry is not critical for short swimming performance.

(C) 2015 Elsevier B.V. All rights reserved.

\section{Introduction}

Due to innate characteristics of the human body a certain level of asymmetry is considered acceptable and estimated to occur in $96 \%$ of the population (Annet, 1988). Although asymmetry is enlarged and considered normal and adaptive in predominately unilateral sports (Saccol et al., 2010), it may weaken performance in cyclic and continuous activities as swimming (Sanders et al., 2012). Theoretically, the application of similar propelling forces from both right and left upper limbs may positively affect swimming performance (i.e., speed) and contribute to a more adequate body position, reducing the hydrodynamic drag (Sanders, Thow, \& Fairweather, 2011). This is even more important for the front crawl technique, as the upper limbs are the major contributors for overall propulsion (Deschodt, Arsac, \& Rouard, 1999).

In fact, when performing front crawl, swimmers intend to keep an uninterrupted application of force in water, leading to a lower intra-cycle velocity variation (Barbosa et al., 2010; Figueiredo, Seifert, Vilas-Boas, \& Fernandes, 2012) and, consequently, to an optimal performance (Formosa, Mason, \& Burkett, 2011). However, the upper limbs alternating movements do not necessarily ensure symmetry, particularly regarding upper limb coordination (Seifert, Chollet, \& Allard, 2005), hand speed (Keskinen, 1994), hand path (Aujouannet, Bonifazi, Hintzy, Vuillerme, \& Rouard, 2006a) and propulsive forces (Formosa, Sayers, \& Burkett, 2013; Formosa et al., 2011; Yeater, Martin, White, \& Gilson, 1981). So, it is not clear if the reported asymmetries can alter the optimal function or simply are within the limits of normal variation. The challenge is to examine till which extent it can be assumed as a coordination mode and not a functional error (Seifert et al., 2005). For instance, high level swimmers have an enhanced control of body roll (Cappaert, Pease, \& Troup, 1995) and a greater medio-lateral hand motion augmenting the potential to develop propulsive forces (Payton, Hay, \& Mullineaux, 1997). Nevertheless, the majority of high level swimmers demonstrate asymmetrical propulsion (Formosa et al., 2013).

One other factor that may increase the asymmetrical propulsion is the breathing action. In fact, it appears that different breathing modes (unilateral vs. bilateral) lead to an asymmetrical arm coordination in the front crawl swimming (Seifert et al., 2005), as breathing causes a lateral movement that disturb the body alignment (Lerda \& Cardelli, 2003). Trying to clarify this issue, previous studies (Seifert, Chehensse, Tourny-Chollet, Lemaitre, \& Chollet, 2008; Seifert et al., 2005; Tourny-Chollet, Seifert, \& Chollet, 2009) showed that handedness, breathing laterality and breathing pattern commonly used, have an effect both in force and coordination symmetry. Experiments aiming to examine how those asymmetries affect swimming performance could help to clarify the swimming science community about the amount of asymmetry that may be considered acceptable.

Swimming velocity is dependent of both propulsive and drag forces, being the ability to obtain high values of propulsive force considered essential to enhance swimming performance (Formosa et al., 2013). Moreover, its importance varies according to the distance to be swum; that is, the importance of forces exerted in water is higher over short distances, while for long distances technical ability plays a major role (Morouço, Keskinen, Vilas-Boas, \& Fernandes, 2011). Despite the current efforts to quantify the exerted forces during swimming, its assessment in ecological conditions (i.e., in free swimming) is vastly complex, if not almost impossible (Sanders \& Psycharakis, 2009). Thus, tethered swimming has been used to estimate swimming propulsion, since this methodology imply a similar use of all body structure (Dopsaj, Matkovic, Thanopoulos, \& Okicic, 2003) and muscle activity pattern 
(Bollens, Annemans, Vaes, \& Clarys, 1988) to free swimming, and has a good test-retest reliability (Kjendlie \& Thorsvald, 2006). Using a load-cell, it is possible to assess individual force to time curves, allowing to analyse and compare the upper limbs cycle profiles (Keskinen, 1997; Morouço et al., 2011) and to diagnosis bilateral upper limb asymmetries in front crawl (dos Santos, Pereira, Papoti, Bento, \& Rodacki, 2013). Finally, tethered swimming does not significantly alter stroke and the physiological responses compared with free swimming suggesting that it can be used to evaluate swimmers (Morouço, Marinho, Keskinen, Badillo, \& Marques, 2014), despite the minor kinematic differences that have been noted (Maglischo, Maglischo, Sharp, Zier, \& Katz, 1984).

The aim of this study was to examine the magnitude of upper limb kinetic asymmetries in front crawl tethered swimming at maximal intensity. Furthermore, we intended to analyse if these asymmetries would affect the relationship of the exerted forces with swimming performance. The first hypothesis was that asymmetric force exertion would occur in the majority of swimmers, independently of their level. Second, it was expected that the ability to exert similar forces with both upper limbs would affect the relationship between tethered forces and swimming velocity, thereby providing evidence of the effects of force symmetry on swimming performance.

\section{Methods}

\subsection{Participants}

Eighteen male swimmers (age $=15.6 \pm 2.1$ years old, height $=1.72 \pm 0.07 \mathrm{~m}$, body mass $=$ $66.3 \pm 9.2 \mathrm{~kg}$ ) volunteered as subjects. Skill level was assessed from the $100 \mathrm{~m}$ freestyle personal best: the mean \pm SD was $59.6 \pm 4.6 \mathrm{~s}$ (min. 52.00 and max. $66.44 \mathrm{~s}$ ), representing various levels of competitive performance. Yet, all participants were sprint or middle-distance specialists. To minimize the effect of the breathing pattern adopted over years of training, only the swimmers with unilateral breathing were considered (Seifert et al., 2008). Swimmers had, at least, 5 years of competitive experience and did not show shoulder injury, and thus we assumed that kinetic asymmetry would not be attributable to impingement (Yanai \& Hay, 2000). Before testing, participants read and signed an informed consent form, approved by the University Ethics Committee and accordingly to the Declaration of Helsinki.

\subsection{Experimental procedures}

After a $1000 \mathrm{~m}$ front crawl moderate intensity warm-up ( $400 \mathrm{~m}$ swim, $100 \mathrm{~m}$ pull, $100 \mathrm{~m}$ kick, $4 \times 50 \mathrm{~m}$ at increasing speed and $200 \mathrm{~m}$ easy swim), each swimmer completed a maximal $30 \mathrm{~s}$ front crawl tethered swimming test. Each subject used a belt attached to the hip by a steel cable (sufficiently inflexible that its elasticity could be neglected) with $3.5 \mathrm{~m}$ length. A load-cell system connected to the cable was used as a measuring device (maximum capacity of $4905 \mathrm{~N}$ ) and the signal was acquired at a sampling rate of $100 \mathrm{~Hz}$. The load-cell was connected to a Globus Ergometer data acquisition system (Globus, Cologne, Italy) that exported the data to a personal computer. Preceding the starting signal, swimmers adopted a horizontal position with the cable fully extended and swam three upper limbs cycles at low intensity. Data collection only started after the first maximum cycle was completed to avoid the inertial effect of the cable extension usually observed immediately before or during the first upper limb action (cf. Morouço et al., 2011). The participants were told to follow the breathing pattern they would normally apply during a $50 \mathrm{~m}$ freestyle event and the end of the test was set through an acoustic signal emitted by the scientific personnel. Prior to data collection, the participants underwent familiarization trials with tethered apparatus.

Twenty-four hours afterwards each participant performed a maximal $50 \mathrm{~m}$ front crawl bout with an underwater start (selected to diminish the effect of start and glide) to obtain the time that was set to be the performance criterion.

For both tests swimmers were able to use their whole-body. It was intended not to neglect the role of the lower limbs and their importance for body coordination, and even propulsion. Participants were verbally encouraged throughout the tests to maintain maximal effort over the total duration of the exercise. 
Breathing laterality was established by determining the preferential breathing side from a questionnaire, as previously suggested (Seifert et al., 2005). After determining if the swimmers used unilateral or bilateral breathing patterns, only swimmers using the unilateral pattern were included in the experiments.

\subsection{Data analysis}

Tethered swimming data was explored using a signal processing software (AcqKnowledge v.3.7, Biopac Systems, Santa Barbara, USA) and filtered with a 4.3-4.8 Hz cut-off low-pass. The selection of the cut-off value was done according to residual analysis (residual error versus cut-off frequency). Individual force to time - $\mathrm{F}(t)$ - curves were assessed and registered to obtain the values of force production per cycle, both for the right and left upper limbs. As the force vector in the tethered system presented a small angle to the horizontal, data was corrected computing the horizontal component of force. The mean of the peak forces along the $30 \mathrm{~s}$ and the maximum peak force were estimated for both upper limbs of each swimmer. Video images were also captured (Casio Exilim EX-F1, Casio, USA), allowing identifying the dominant ${ }_{(\mathrm{d}}$ ) (and non-dominant $\left({ }_{n d}\right)$ ) upper limb according to the higher mean of the peak forces in the first five cycles. The peak force on each cycle was assessed for both upper limbs, allowing calculating the slope of peak forces along the bout. Finally, symmetry index (SI) was calculated according to the following equation (Robinson, Herzog, \& Nigg, 1987):

$$
\operatorname{SI}(\%)=\frac{X_{\mathrm{d}}-X_{\mathrm{nd}}}{1 / 2\left(X_{\mathrm{d}}+X_{\mathrm{nd}}\right)} \times 100
$$

where $X_{\mathrm{d}}$ and $X_{\mathrm{nd}}$ are the mean of the peak forces recorded for the dominant and non-dominant upper limbs, respectively. As suggested by the referred authors, $-10 \%<\mathrm{SI}<10 \%$ indicates symmetry, whereas $\mathrm{SI}<-10 \%$ and $\mathrm{SI}>10 \%$ indicates asymmetry. The swimming velocity ( $v 50)$ was assessed by the ratio between the test distance and the corresponding chronometric time.

\subsection{Statistical procedures}

Variables were expressed as means and standard deviations. After normality and homoscedasticity assumption were checked (Shapiro-Wilk and Levene tests, respectively), dependent and independent $t$-tests were performed between dominant and non-dominant upper limbs, and participants, respectively. Linear regression was computed to estimate the slope of peak forces along the $30 \mathrm{~s}$ swim for each upper limb. Partial correlations between tethered forces and swimming performance controlling the effect of the symmetry index were performed. Statistical procedures were performed using SPSS 20.0 for Windows ${ }^{\circledR}$ (Chicago, IL). Post hoc analysis of power $(1-\beta)$ and the values of Cohen's d effect size for repeated measures (ES) were accomplished using the G-Power 3.1.9.2 for Windows ${ }^{\circledR}$ (University of Kiel, Germany). The level of statistical significance was set at $p<0.05$.

\section{Results}

In Table 1 it is possible to observe the mean \pm SD values of the variables obtained in the $30 \mathrm{~s}$ tethered test. Both mean and maximum peak forces were superior for the dominant upper limb, with the non-dominant upper limb presenting a lower decrease along the maximal bout. The $50 \mathrm{~m}$ free

Table 1

Mean \pm SD values of tethered swimming variables for the dominant and non-dominant upper limbs. P-values, effect size (ES), and power analysis values $(1-\beta)$ are presented $(n=18)$.

\begin{tabular}{lllrrr}
\hline & Dominant upper limb & Non-dominant upper limb & $P$-value & ES & $1-\beta$ \\
\hline Mean peak forces (N) & $211.2 \pm 30.5$ & $175.7 \pm 32.8$ & $<0.001$ & 1.46 & 0.99 \\
Maximum peak forces (N) & $271.9 \pm 28.7$ & $217.0 \pm 29.3$ & 0.018 & 0.61 & 0.69 \\
Slope of forces $\left(\mathrm{N} \mathrm{s}^{-1}\right)$ & $-3.84 \pm 1.65$ & $-2.51 \pm 1.02$ & $<0.001$ & 2.77 & 1.00 \\
\hline
\end{tabular}




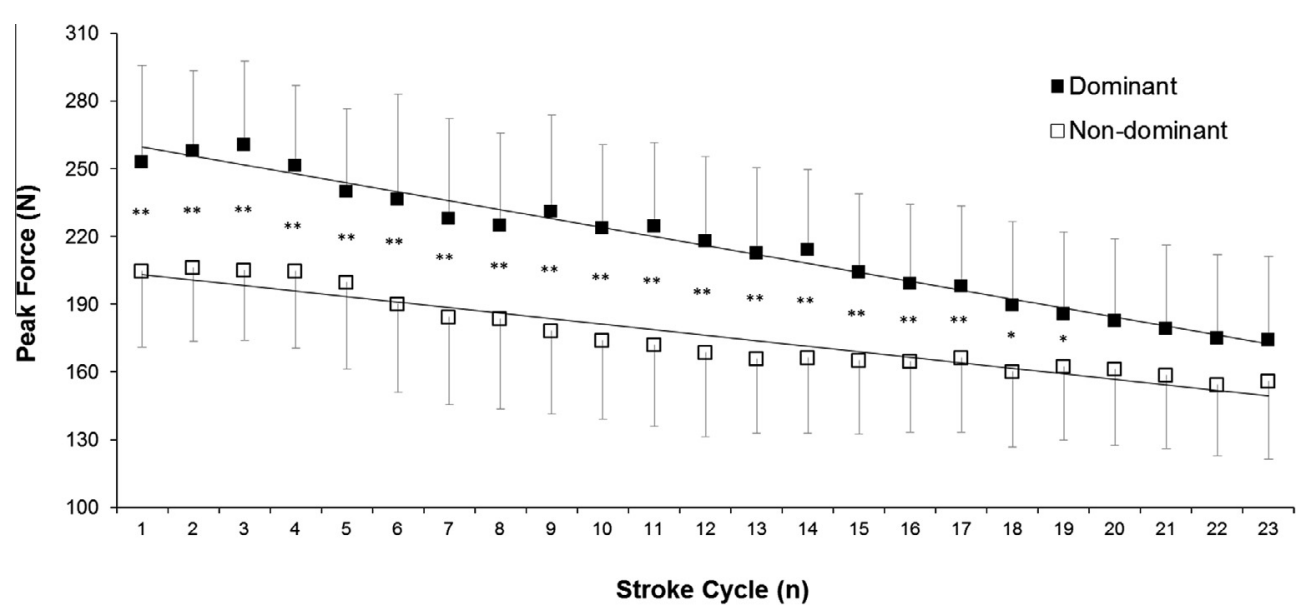

Fig. 1. Mean \pm SD values of peak force per cycle with corresponding linear regression for dominant and non-dominant upper limbs. ${ }^{*}(p<.05)$ and ${ }^{* *}(p<.01)$ lower than dominant upper limb.

\section{Table 2}

Partial correlations between swimming performance and tethered swimming variables, controlling the effect of the symmetry index.

\begin{tabular}{lrc}
\hline & \multicolumn{2}{l}{ Swimming performance } \\
\cline { 2 - 3 } & $r$ & $p$ \\
\hline Mean peak forces dominant upper limb & -0.92 & $<0.001$ \\
Mean peak forces non-dominant upper limb & -0.89 & $<0.001$ \\
Maximum peak forces dominant upper limb & -0.82 & $<0.001$ \\
Maximum peak forces non-dominant upper limb & -0.84 & $<0.001$ \\
Slope of forces dominant upper limb & 0.13 & 0.613 \\
Slope of forces non-dominant upper limb & 0.04 & 0.890 \\
\hline
\end{tabular}

swimming performance ranged between 25.6 and $30.9 \mathrm{~s}$, with a mean of $28.6 \pm 1.75 \mathrm{~s}$. Symmetry index mean \pm SD was $19.0 \pm 14.0$ (range: $3.3-48.5 \%$ ), leading to an asymmetrical force exertion in twelve of the studied swimmers (66.7\%). The applied questionnaire showed that all of these twelve swimmers presented a breathing laterality opposite to the side of the force asymmetry. No differences were found between these swimmers and swimmers with force symmetry, both in tethered swimming variables and in swimming performance.

The mean \pm SD values of peak force per cycle with corresponding linear regression for dominant and non-dominant upper limbs are displayed in Fig. 1.

Table 2 presents the partial correlation between the tethered swimming variables and free swimming performance. There were negative and meaningful associations when controlling isolated the effect of the symmetry index. Last, partial correlation indicated a very strong relationship between the dominant and non-dominant mean forces $(r=0.99, p<0.001)$.

\section{Discussion}

This study aimed to examine front crawl upper limbs asymmetries during maximal tethered swimming. It was observed that the majority of swimmers (66.7\%) presented an asymmetrical force exertion between upper limbs, confirming the first hypothesis. Furthermore, the symmetry index stood up as factor to control in the relationships between exerted forces and swimming performance, confirming the second hypothesis. 
The assessment of biomechanical asymmetries is useful in both clinical and research settings, being possible to characterize the functional imbalance between upper limbs with a single discrete measure (Evershed, Burkett, \& Mellifont, 2014). Theoretically, this functional imbalance could augment the front crawl intra-cycle velocity variation, increase the energy cost for the same average speed (Figueiredo et al., 2012) and deteriorate body postures by maximising hydrodynamic drag (Sanders et al., 2012). In the current study, the majority of the participants presented force asymmetry and there was a large inter-individual variability, with symmetry indexes ranging from $3.3 \%$ to $48.5 \%$ confirming our first hypothesis. These results agree with some of the previous literature (Formosa et al., 2013) and highlight the predictable force imbalance, over the course of repeated crawl movements and years of training (Tourny-Chollet et al., 2009). Over time, unilateral breathing and the development of greater strength of the dominant upper limb may lead to a differentiation in the swimmers ability to exert balanced forces. This is even more usual in sprint specialists, who have a more brutal closure of the open kinetic chain at the end of arm recovery and compete with higher stroke rates (Pelayo, Sidney, Kherif, Chollet, \& Tourny, 1996).

It has been suggested that the dominant limb might be used mainly for propulsion and the nondominant for control and support (Seifert et al., 2005). If so, temporal asymmetry could reflect the variability of impulse, i.e., the force-time pattern (Aujouannet, Rouard, \& Bonifazi, 2006b). Although it is not accurate to estimate the impulse per upper limb cycle, as swimmers tend to use overlapping coordination in high intensity exercises (Figueiredo et al., 2012), the higher forces (mean and maximum) obtained by the dominant upper limb in the current study confirm the referred suggestion. Complementarily, we were interested in examining how the force (a)symmetry develops along a maximal bout. A deeper analyses showed that higher force values were more noticeable in the first cycles of the bout (cf. Fig. 1), suggesting that some previous studies underestimated the level of asymmetry, as they used only two cycles (Aujouannet et al., 2006a) or the mean values of the overall effort (dos Santos et al., 2013). Thus, the proposed methodology, i.e., the evaluation of asymmetry throughout the exercise as a complement to a single discrete measure, may bring new insights about the force pattern in short duration and high intensity bouts. Accordingly, the usage of semi-tethered swimming would suit the possibility to examine the impact that force asymmetry has on other behavioural and performance indicators (e.g. intra-cycle velocity variation).

Lastly, the first hypothesis can be confirmed by the total correspondence of breathing laterality opposite to the side of the force asymmetry. Both the body alignment as well as the continuity of propulsive actions can be disturbed by the breathing movements (Lerda \& Cardelli, 2003). In fact, swimmers with extensive years of training using this breathing pattern are more likely to develop coordination asymmetry between upper limbs (Seifert et al., 2005). The current study does not come up with a definite solution about the origin of these asymmetries; nevertheless it does provide some valuable evidences in terms of their importance for sprint swimming performance. In fact, they can be due to pre-existing strength deficits, limb dominance or even differences on technique (Evershed et al., 2014; Sanders et al., 2012). Even so, the assessment of propulsive forces through in-water experiments may be a useful tool for imbalance diagnosis, and ultimately to provide coaches additional information for training prescription.

Seifert et al. $(2008,2005)$ and Tourny-Chollet et al. (2009) showed that both force and coordination symmetry are affected by handedness, breathing laterality and breathing pattern commonly used. Accordingly, we were interested in understanding how force asymmetry would relate with swimming performance.

Tethered swimming may be a proper methodology to measure the maximum force that, theoretically, corresponds to the propulsion produced to overcome the water resistance at maximum free swim velocity (Morouço et al., 2011, 2014). However, this methodology requires the participants to be familiarized with the apparatus (Psycharakis, Paradisis, \& Zacharogiannis, 2011). In the week prior to the experiments of the present study, several trials were conducted aiming that familiarization. Although the velocity of displacement cannot be related between tests, the remaining option is to make the data collection at maximal intensity and thereafter control for the effect of significant variables (i.e., compute partial correlations). Our results underlined the role that force asymmetry plays in swimming performance, suggesting that, at least for short distances, a certain amount of asymmetry is not critical. This is coherent with the assumption that maximal velocity is more dependent on pulling 
force characteristics that on technical and energetic abilities of swimmers (Dopsaj et al., 2003). Nevertheless these results were obtained with fully tethered swimming. Thus, it does not come up with a definite solution to isolate the contribution of force for swimming performance. Indeed, further studies using semi-tethered swimming may supplement the presented analysis with associations of force asymmetry with intra-cyclic velocity variations and inter-arm coordination.

Previous investigations on the relationship between the ability to exert force in the water and swimming velocity (Keskinen, 1994; Morouço et al., 2011, 2014; Yeater et al., 1981) have pointed that force is a major factor to consider in short distance events. Those authors have used different approaches (regarding time and distances), but consistently showed moderate-to-strong associations between tethered forces and swimming performance. In the present study, similar associations were obtained. However, those associations revealed higher magnitudes when controlling isolated the effect of symmetry index thus confirming the second hypothesis. Opposing to dos Santos et al. (2013), a higher force asymmetry did not lead to a worst performance. These authors disregarded the overlap between right and left upper limbs, did not use a symmetry index and omitted the inherent lateral dominance, requiring caution when interpreting their results. In fact, to some extent, force asymmetry may benefit the dominant upper limb to apply a better impulse of force (Seifert et al., 2005). Aiming to enhance swimming performance over short distances, force symmetry should only be minimized if it causes too much disturbance to performance.

\section{Conclusion}

The contribution of force exertion on swimming performance has long been discussed and suggests that the force exerted in water is a major factor for success. However, this is the first study examining those associations taking in consideration the inherent force asymmetry. The majority of the studied swimmers (66.7\%) presented an asymmetrical force exertion, i.e., an absolute symmetry index higher than $10 \%$. The higher force asymmetry did not lead to worst swimming performance, but had a significant effect on the latter. Thus, to some extent, force asymmetry may not be critical to achieve high swimming velocities. A deeper analysis revealed that force asymmetry is most due to different force exertion in the first cycles of a maximal bout.

\section{Acknowledgments}

The authors wish to thank Henrique Neiva for his useful help during data collection and to all the swimmers and coaches for their collaboration. This investigation was supported by grants of the Portuguese Science and Technology Foundation (SFRH/BD/66910/2009) and University of Beira Interior (UBI/FCSH/Santander/2010).

\section{References}

Annet, M. (1988). Handedness and cerebral dominance: the right shift theory. The Journal of Neuropsychiatry and Clinical Neurosciences, 10, 459-469.

Aujouannet, Y. A., Bonifazi, M., Hintzy, F., Vuillerme, N., \& Rouard, A. H. (2006a). Effects of a high-intensity swim test on kinematic parameters in high-level athletes. Applied Physiology, Nutrition, and Metabolism, 31, 150-158.

Aujouannet, Y. A., Rouard, A. H., \& Bonifazi, M. (2006b). Effects of fatigue on the kinematic hands symmetry in freestyle. Portuguese Journal of Sport Sciences, 6, 24-26.

Barbosa, T. M., Bragada, J. A., Reis, V. M., Marinho, D. A., Carvalho, C., \& Silva, A. J. (2010). Energetics and biomechanics as determining factors of swimming performance: updating the state of the art. Journal of Science and Medicine in Sport, 13, 262-269.

Bollens, E., Annemans, L., Vaes, W., \& Clarys, J. P. (1988). Peripheral EMG comparison between fully tethered and free front crawl swimming. In B. Ungerechts, K. Wilke, \& K. Reischle (Eds.), Swimming Science V (pp. 173-181). London: Spon Press.

Cappaert, J. M., Pease, D. L., \& Troup, J. P. (1995). Three-dimensional analysis of the men's 100-m freestyle during the 1992 Olympic games. Journal of Applied Biomechanics, 11, 103-112.

Deschodt, V. J., Arsac, L. M., \& Rouard, A. H. (1999). Relative contribution of upper limbs and legs in humans to propulsion in 25m sprint front-crawl swimming. European Journal of Applied Physiology, 80, 192-199.

Dopsaj, M., Matkovic, I., Thanopoulos, V., \& Okicic, T. (2003). Reliability and validity of basic kinematics and mechanical characteristics of pulling force in swimmers measured by the method of tethered swimming with maximum intensity of 60 seconds. Physical Education \& Sport, 1, 11-22. 
dos Santos, K. B., Pereira, G., Papoti, M., Bento, P. C., \& Rodacki, A. (2013). Propulsive force asymmetry during tetheredswimming. International Journal of Sports Medicine, 34, 606-611.

Evershed, J., Burkett, B., \& Mellifont, R. (2014). Musculoskeletal screening to detect asymmetry in swimming. Physical Therapy in Sport, 14, 33-38.

Figueiredo, P., Seifert, L., Vilas-Boas, J. P., \& Fernandes, R. J. (2012). Individual profiles of spatio-temporal coordination in high intensity swimming. Human Movement Science, 31, 1200-1212.

Formosa, D. P., Mason, B., \& Burkett, B. (2011). The force-time profile of elite front crawl swimmers. Journal of Sports Sciences, 29, 811-819.

Formosa, D. P., Sayers, M. G. L., \& Burkett, B. (2013). Front-crawl stroke-coordination and symmetry: a comparison between timing and net drag force protocols. Journal of Sports Sciences, 31, 759-766.

Keskinen, K. L. (1994). Measurement of technique in front crawl swimming. In M. Miyashita, Y. Mutoh, \& A. B. Richardson (Eds.), Medicine and Science in Aquatic Sports (pp. 117-125). Basel: Karger.

Keskinen, K. L. (1997). Evaluation of technique performances in freestyle swimming. Kinesiology, 2, 30-38.

Kjendlie, P. L., \& Thorsvald, K. (2006). A tethered swimming power test is highly reliable. Portuguese Journal of Sport Sciences, 6 , $231-233$.

Lerda, R., \& Cardelli, C. (2003). Breathing and propelling in crawl as a function of skill and swim velocity. International Journal of Sports Medicine, 24, 75-80.

Maglischo, C., Maglischo, E., Sharp, R., Zier, D., \& Katz, A. (1984). Tethered and nontethered crawl swimming. In J. Terauds, K. Barthels, E. Kreighbaum, R. Mann, \& J. Crakes (Eds.), Proceedings of ISBS: Sports Biomechanics (pp. 163-176). Del Mar: Academic Publication.

Morouço, P. G., Keskinen, K. L., Vilas-Boas, J. P., \& Fernandes, R. J. (2011). Relationship between tethered forces and the four swimming techniques performance. Journal of Applied Biomechanics, 27, 161-169.

Morouço, P. G., Marinho, D. A., Keskinen, K. L., Badillo, J. J., \& Marques, M. C. (2014). Tethered swimming can be used to evaluate force contribution for short-distance swimming performance. Journal of Strength and Conditioning Research, 28, 3093-3099.

Payton, C. J., Hay, J. G., \& Mullineaux, D. R. (1997). The effect of body roll on hand speed and hand path in front crawl swimming - a simulation study. Journal of Applied Biomechanics, 13, 300-315.

Pelayo, P., Sidney, M., Kherif, T., Chollet, D., \& Tourny, C. (1996). Stroking characteristics in freestyle swimming and relationships with anthropometric characteristics. Journal of Applied Biomechanics, 12, 197-206.

Psycharakis, S. G., Paradisis, G. P., \& Zacharogiannis, E. (2011). Assessment of accuracy, reliability and force measurement errors for a tethered swimming apparatus. International Journal of Performance Analysis in Sport, 11, 411-416.

Robinson, R. O., Herzog, W., \& Nigg, B. M. (1987). Use of force platform variables to quantify the effects of chiropractic manipulation on gait symmetry. Journal of Manipulative and Physiological Therapeutics, 10, 172-176.

Saccol, M. F., Gracitelli, G. C., da Silva, R. T., Laurino, C. F., Fleury, A. M., Andrade, M. S., et al (2010). Shoulder functional ratio in elite junior tennis players. Physical Therapy in Sport, 11, 8-11.

Sanders, R. H., \& Psycharakis, S. G. (2009). Rolling rhythms in front crawl swimming with six-beat kick. Journal of Biomechanics, $42,273-279$.

Sanders, R. H., Thow, J., \& Fairweather, M. M. (2011). Asymmetries in Swimming: Where Do They Come from? Journal of Swimming Research, 18, 1-11.

Sanders, R., Thow, J., Alcock, A., Fairweather, M., Riach, I., \& Mather, F. (2012). How can asymmetries in swimming be identified and measured? Journal of Swimming Research, 19, 1-15.

Seifert, L., Chollet, D., \& Allard, P. (2005). Upper limb coordination symmetry and breathing effect in front crawl. Human Movement Science, 24, 234-256.

Seifert, L., Chehensse, A., Tourny-Chollet, C., Lemaitre, F., \& Chollet, D. (2008). Effect of breathing pattern on arm coordination symmetry in front crawl. Journal of Strength and Conditioning Research, 22, 1670-1676.

Tourny-Chollet, C. T., Seifert, L., \& Chollet, D. (2009). Effect of force symmetry on coordination in crawl. International Journal of Sports Medicine, 30, 182-187.

Yanai, T., \& Hay, J. G. (2000). Shoulder impingement in front crawl swimming: I. A method to identify impingement. Medicine E' Science in Sports \& Exercise, 32, 21-29.

Yeater, R. A., Martin, R. B., White, M. K., \& Gilson, K. H. (1981). Tethered swimming forces in the crawl, breast and back strokes and their relationship to competitive performance. Journal of Biomechanics, 14, 527-537. 